\title{
Microwave-assisted Synthesis of Hierarchical ZnO Nanostructures and Their Photocatalytic Properties
}

\author{
Ming $\mathrm{OU}^{1}$, Lin $\mathrm{MA}^{1, *}$, Limei XU${ }^{1}$, Haizhen $\mathrm{LI}^{1}$, Zhuomei YANG ${ }^{1}$ and Zhifeng $\mathrm{LAN}^{1}$ \\ ${ }^{1}$ School of Chemistry and Chemical Engineering, Institute of Physical Chemistry, Lingnan Normal \\ University, Zhanjiang 524048, P. R. China
}

\begin{abstract}
Hierarchical $\mathrm{ZnO}$ nanostructures were fabricated via a rapid and facile microwave-assisted route with different zinc salts as reactants. The obtained hierarchical $\mathrm{ZnO}$ nanostructures have good crystallinity and high purity. Moreover, it was found that various zinc salts have an obvious effect on the morphologies and microstructures of the final products. Additionally, the photocatalytic activity of the obtained $\mathrm{ZnO}$ samples under visible-light irradiation was also evaluated by degradation of Rhodamine B (RhB).
\end{abstract}

\section{Introduction}

Recently, hierarchical micro/nanostructures, which are assembled from nanoparticles, nanorods or nanowires, and nanosheets or nanoplates as building blocks, have attracted significant attention based on the supposition that the hierarchical structures could inherit the unique advantages of their pristine building blocks and obtain intriguing properties from their superior secondary architecture ${ }^{1,2}$. Specially, it has been demonstrated that the hierarchical architecture constructed by $2 \mathrm{D}$ nanosheets results in superior photocatalytic performance, as the porous structures among the nanosheets are favorable for efficient reactants transport and photo-energy harvesting ${ }^{3,4}$.

Zinc oxide $(\mathrm{ZnO})$, with a wide band gap $(3.37 \mathrm{eV})$, has witnessed an explosion of interest in the past few years because of advances in synthesis and unique optoelectronic, photochemical, and catalytic properties ${ }^{5-8}$. As one of the most important semiconductor photocatalysts, $\mathrm{ZnO}$ has attracted considerable interest owing to its high photosensitivity and stability $^{9,10}$. To date, synthesis of $\mathrm{ZnO}$ hierarchical nanostructures have drawn extensive research attention due to their porous structured networks and high internal surface area which result in the enhancement of optical, electrical and catalytic properties. A variety of typical methods have been reported to construct $\mathrm{ZnO}$ hierarchical micro/nanostructures including chemical vapor deposition ${ }^{11,12}$, pulsed laser deposition ${ }^{13,14}$, spray pyrolysis ${ }^{15,16}$, electrochemical deposition ${ }^{17,18}$, liquid reflux ${ }^{19}$, sonochemical method ${ }^{20,21}$ as well as hydrothermal or solvothermal approach. Amongst these, hydrothermal or solvothermal

*Corresponding author:ml_buct@126.com 
approach becomes the most commonly used liquid-phase synthetic route to obtain different hierarchical $\mathrm{ZnO}$ nanostructures ${ }^{22-28}$. Moreover, these routes usually involve time-consuming and high-temperature processes and thus definitely hinder further study of these nanomaterials.

Currently, microwave-assisted heating technique based on volumetric nature of the power dissipation in a dielectric is particularly attractive in inorganic nanomaterials preparation. Compared with the traditional hydrothermal method, the microwave-assisted irradiation method has many advantages, such as kinetic enhancement, low reaction temperature, short reaction period, easy control of the overall particle size and effectual avoidance of aggregation process and so on ${ }^{29,30}$. Up to now, various hierarchical $\mathrm{ZnO}$ nanostructures have been successfully prepared by a microwave-related method ${ }^{31-33}$. Herein, we report an efficient and simple approach for synthesis of hierarchical $\mathrm{ZnO}$ nanostructures by a rapid microwave-assisted method with different zinc salts as reactants.

\section{Experimental Procedure}

\subsection{Microwave-assisted synthesis of hierarchical $\mathrm{ZnO}$ nanostructures}

In a typical synthesis, $3 \mathrm{mmol}$ zinc nitrate and $4 \mathrm{mmol}$ polassium sodium citrate were dissolved in $60 \mathrm{ml}$ deionized water under violent stirring. Then $15 \mathrm{mmol} \mathrm{NaOH}$ was rapidly poured into the above solution. After stirring for $10 \mathrm{~min}$, the above mixture was finally transferred into a $100 \mathrm{ml}$ round flask and placed in a microwave oven $(650 \mathrm{~W}, 2.45 \mathrm{GHz})$ with a refluxing apparatus. The mixture was heated by microwave irradiation for $20 \mathrm{~min}$ at $90{ }^{\circ} \mathrm{C}$ under refluxing. The resulting product was collected by centrifuge and washed three times using deionized water and absolute ethanol, then dried in vacuum at $60^{\circ} \mathrm{C}$ for $12 \mathrm{~h}$. For convenience, the obtained $\mathrm{ZnO}$ sample was termed as $\mathrm{N}-\mathrm{ZnO}$. In addition, zinc sulphate and zinc acetate were also used to prepare $\mathrm{ZnO}$ by a similar synthetic route and the products were denoted as $\mathrm{S}-\mathrm{ZnO}$ and $\mathrm{A}-\mathrm{ZnO}$, respectively.

\subsection{Characterization of $\mathrm{ZnO}$ samples}

$\mathrm{XRD}$ analysis was performed on a D/Max-2550 X-ray diffractometer with monochromatized $\mathrm{CuK} \alpha$ radiation $(\lambda=0.1540562 \mathrm{~nm})$. SEM images were taken with FEI SIRION-100 field-emission scanning electron microscope. Absorption spectrum was measured on a UV-vis spectrophotometer (UV-2550) in the wavelength range of 200-700 nm.

\subsection{Photocatalytic test of $\mathrm{ZnO}$ samples}

Photocatalytic properties of the as-synthesized products were tested by the reduction of aqueous $\mathrm{Cr}(\mathrm{VI})$ and degradation of $\mathrm{RhB}$ solution under visible-light irradiation $(\lambda>420 \mathrm{~nm})$ with a $300 \mathrm{~W}$ Xe lamp, respectively. The catalysts and the $\mathrm{Cr}(\mathrm{VI})$ or $\mathrm{RhB}$ solution were placed in a $400 \mathrm{~mL}$ quartz beaker with stirring and refluxing equipment. The distance between the lamp source and the sample is kept as $30 \mathrm{~cm}$. Prior to illumination, $200 \mathrm{~mL}$ of 10 $\mathrm{mg} / \mathrm{L} \mathrm{RhB}$ aqueous solution containing $100 \mathrm{mg}$ of photocatalyst was magnetically stirred in dark for $60 \mathrm{~min}$ to ensure the adsorption-desorption equilibrium between photocatalyst and $\mathrm{RhB}$. During illumination, about $2 \mathrm{~mL}$ of suspension was taken from the reactor at a scheduled interval and centrifuged at $12000 \mathrm{rpm}$ for $10 \mathrm{~min}$, and the concentration of RhB in 
the supernatant was analyzed by measuring the absorbance at $553 \mathrm{~nm}$ with a Shimadzu UV2550 spectrophotometer, respectively.

\section{Results and Discussions}
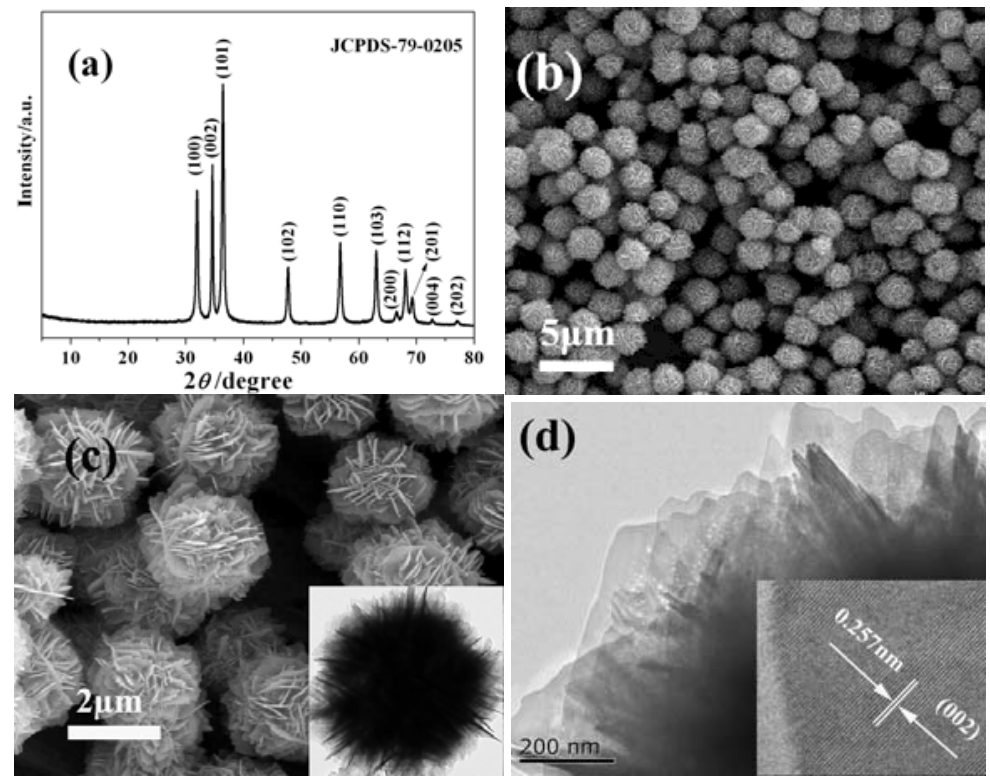

Fig. 1 (a) XRD patterns, (b-d) SEM and TEM images of the $\mathrm{N}-\mathrm{ZnO}$ microflowers prepared by a microwave-assisted route.

Fig. 1a depicts the XRD pattern of the obtained N-ZnO sample, which shows that the major detectable diffraction peaks can be easily attributed to the pure hexagonal phase of $\mathrm{ZnO}$, well matching with the standard powder diffraction of ZnO (JCPDS 79-0205). No impurity phase can be found, which confirms the high purity of the samples. The high and sharp peaks indicate that the $\mathrm{N}-\mathrm{ZnO}$ samples are well crystallized despite the slightly broadened peaks arising from the nanostructured phases. Fig. 1b provides a typical SEM image of the $\mathrm{N}-\mathrm{ZnO}$ samples, which clearly displays a well-dispersed distribution of sphere-like particles with uniform sizes and high yields. It is estimated that $\mathrm{N}-\mathrm{ZnO}$ particles have an average diameter of $2.32 \mu \mathrm{m}$. The surface features of these sphere-like particles were carefully inspected by a higher-resolution SEM image as shown in Fig. 1c. It can be seen that the surfaces of these sphere-like particles are quite rough and covered with numerous interconnected nanosheets. The inserted TEM image of Fig. 1c clearly displays a well-defined microflower with many nanosheets radially stretching out of the edges. The microstructures of the $\mathrm{N}-\mathrm{ZnO}$ were further investigated by HRTEM techniques. The HRTEM image (inset in Fig. 1d) shows lattice fringes with d-spacing of $0.257 \mathrm{~nm}$, in agreement with the (002) crystal planes of hexagonal $\mathrm{ZnO}$. According to TEM and SEM observations, it is suggested that these $\mathrm{N}-\mathrm{ZnO}$ microflowers are assembled by numerous nanosheets in a certain way. 

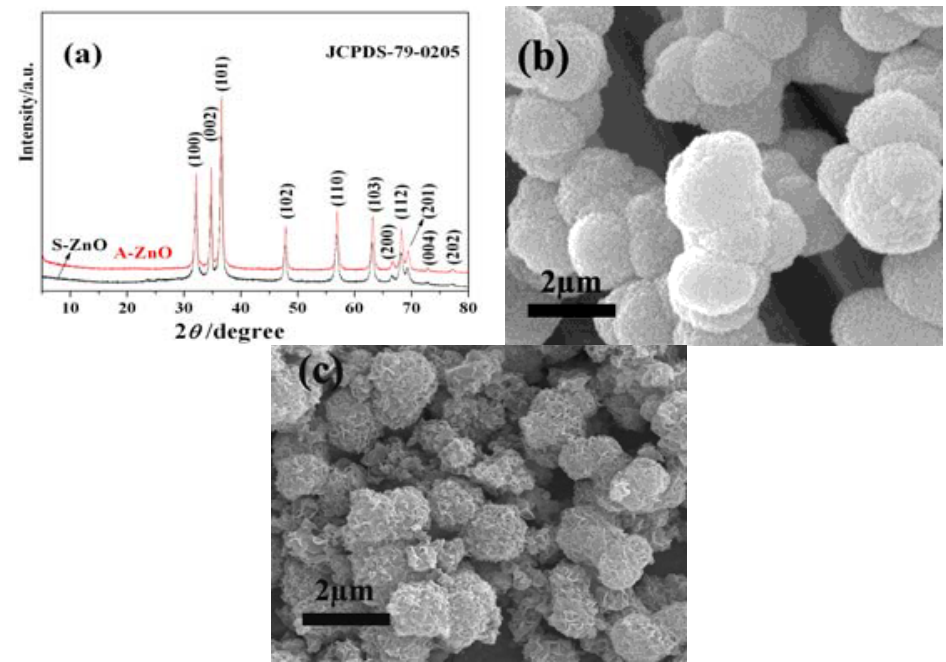

Fig. 2 (a) XRD patterns and SEM images of (b) S-ZnO and (c) A-ZnO samples prepared by a microwave-assisted route.

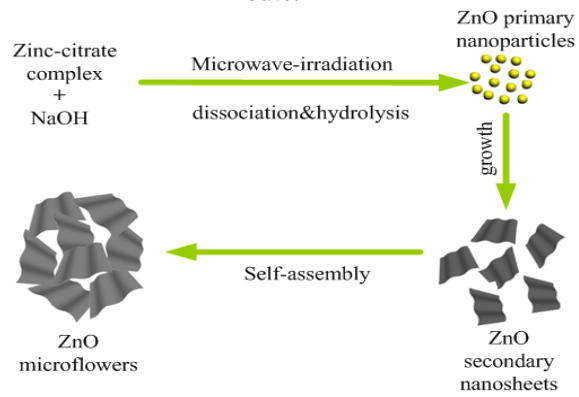

Fig. 3 Illustration of the formation process of the $\mathrm{N}-\mathrm{ZnO}$ microflowers prepared by a microwave-assisted route.

Control experiments were also conducted to investigate the effect of different zinc salts on the morphology and structure of $\mathrm{ZnO}$ products. When zinc sulphate and zinc acetate are adopted as reactants, it can be seen from Fig. 2a that the major detectable diffraction peaks of the obtained products are still indexed to the hexagonal phase of $\mathrm{ZnO}$ (JCPDS 79-0205). Fig. $2 \mathrm{~b}-\mathrm{c}$ display the morphologies and microstructures of the $\mathrm{A}-\mathrm{ZnO}$ and $\mathrm{S}-\mathrm{ZnO}$ products, respectively. It is interesting that both $\mathrm{A}-\mathrm{ZnO}$ and $\mathrm{S}-\mathrm{ZnO}$ display completely different morphology from that of $\mathrm{N}-\mathrm{ZnO}$. The $\mathrm{S}-\mathrm{ZnO}$ samples are cross-linked microspheres as shown in Fig. 2b. Meanwhile, it can be observed that the surfaces are covered with many nanoparticles, meaning that the $\mathrm{S}-\mathrm{ZnO}$ microspheres are constructed with nanoparticles. As for the A-ZnO samples, the ununiform microflowers composed of nanosheets can be detected in Fig. 2c. The above results indicate that the various zinc salts have a remarkble influence on the morphology and microstructure of the final products. Additionally, N2 adsorption-desorption measurements were performed to estimate the surface areas of the obtained products. It was calculated that surface areas of the $\mathrm{N}-\mathrm{ZnO}, \mathrm{A}-\mathrm{ZnO}$ and $\mathrm{S}-\mathrm{ZnO}$ were about $38.08,29.71$ and $17.96 \mathrm{~m}^{2} / \mathrm{g}$, respectively. The plausible formation process of the $\mathrm{N}-\mathrm{ZnO}$ microflowers are preliminarily illustrated in Fig. 3. 

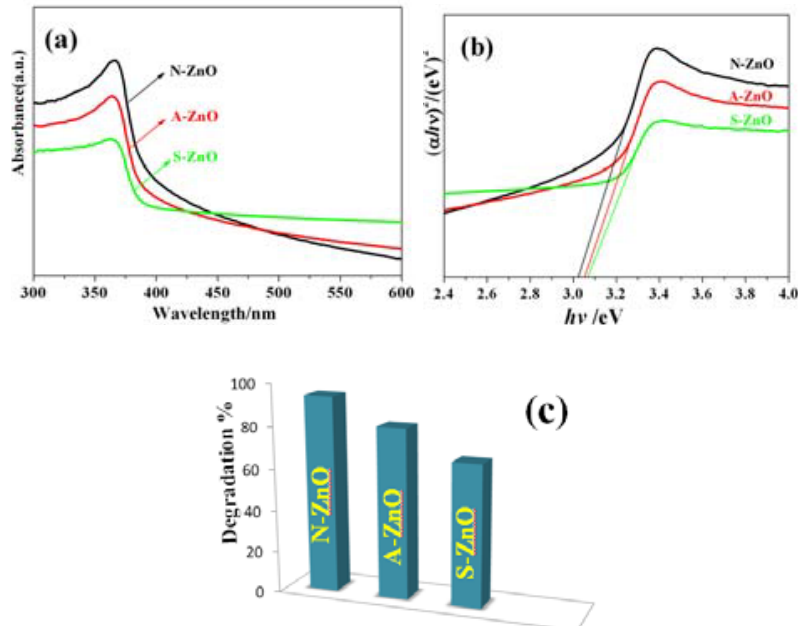

Fig. 4 (a) UV-vis diffuse reflectance spectra (a), (b) the calculation diagram of their bandgaps and (c) column diagram of photocatalytic degradation of $\mathrm{RhB}$ under the visible light irradiation.

Based on the experimental results as well as previous reports ${ }^{22,34,35}$, the probable formation process of the varied $\mathrm{ZnO}$ nanostructures prepared with different zinc salts in our current experiment can be explained as follows. As an efficient chelator for metal ions, it was easy for polassium sodium citrate to chelate $\mathrm{Zn}^{2+}$ to form $\mathrm{Zn}$-citrate complexes. Under microwave rapid heating conditions, $\mathrm{Zn}^{2+}$ ions were continuously supplied at an appropriate rate by dissociation of the complexes. The dissociated $\mathrm{Zn}^{2+}$ ions could react with $\mathrm{OH}^{-}$to form $\mathrm{ZnO}$ primary colloidal nanoparticles. At the same time, the different anions in solution such as nitrate, sulphate and acetate ions could be adsorbed on the surfaces of particles in different ways, which could lead to the growth of primary crystals in different ways, and finally result in different shapes of secondary particles. Afterwards, these secondary particles aggregated together to minimize their surface energy in an oriented way and further formed the final products. The actual growth mechanism of these nanostructures need be further investigated.

Fig. 4a shows the UV-vis absorption spectra of the different $\mathrm{ZnO}$ products prepared with different zinc salts via a microwave irradiation process, which displays the photoabsorption property from the UV light region to the visible light region. Moreover, slight red shift of the absorption edge can also be discerned in the sequence of $\mathrm{S}-\mathrm{ZnO}, \mathrm{A}-\mathrm{ZnO}$ and $\mathrm{N}-\mathrm{ZnO}$. From the curve of the $(\alpha h v) 2$ vs. photo energy (hv) in Fig. $4 \mathrm{~b}$, the band gaps are estimated to be about $3.03 \mathrm{eV}, 3.06 \mathrm{eV}$ and $3.08 \mathrm{eV}$, corresponding to the N-ZnO, A-ZnO and $\mathrm{S}-\mathrm{ZnO}$ products, respectively, which are lightly lower than those of $\mathrm{ZnO}$ materials reported previously 23-26. The photocatalytic degradation activities of the $\mathrm{ZnO}$ products are shown in Fig. 4c. After 120-min degradation process, the degradation rate for Rhodamine B are $93.63 \%, 82.35 \%$ and $64.66 \%$, respectively for the $\mathrm{N}-\mathrm{ZnO}, \mathrm{A}-\mathrm{ZnO}$ and $\mathrm{S}-\mathrm{ZnO}$ products. Amongst the three products, $\mathrm{N}-\mathrm{ZnO}$ exhibits the best photocatalytic performance for Rhodamine B degradation, which might be attributed to the larger surface area and relatively smaller band gap than the other two samples.

\section{Conclusions}

Hexagonal $\mathrm{ZnO}$ hierarchical nanostructures with various morphologies have been synthesized via a facile rapid microwave irradiation route. The results revealed that the morphologies and microstructures of the final products were tremendously influenced by the different zinc salts. In addition, when photocatalytic degradation of $\mathrm{RhB}$, the $\mathrm{Z}-\mathrm{ZnO}$ product 
demonstrated the best visible-light photocatalytic activity which possibly resulted from its relatively large aspect surface areas, narrow band gap, uniform morphologies and sizes. The strategy presented in this work may be feasible to develop into the scale-up production of hierarchical structures.

\section{Acknowledgement}

This work is financially supported by the Guangdong Natural Science Foundation for Joint Training Innovative Talents in East-West-North Guangdong (2014A030307030), the Science and Technology Planning Project of Guangdong Province (2014A010106032), the Stong Innovation School of Engineering Program of Department of Education of Guangdong Province (Distinctive Innovation Project No. 2014KTSCX157), Natural Science Foundation of Lingnan Normal University (LZL1402, LZL1502) and Training Plan to Innovation and Enterprise of Undergraduates of Guangdong Province(201510579257).

\section{References}

1. J.Y. Lao, J.G. Wen, Z.F. Ren. Nano. Lett. 2 (2002) 1287-1290.

2. H.B. Yao, H.Y. Fang, X.H. Wang, S.H. Yu. Chem. Soc. Rev. 40 (2011) 3764-3766.

3. F. Lu, W. Cai, Y. Zhang. Adv. Funct. Mater. 18 (2008) 1047-1052.

4. D. Kim, Y.D. Huh. Mater. Lett. 65 (2011) 2100-2104.

5. M. Saleem, L. Fang, S.F. Shaukat, M.A. Ahmad, R. Raza, M.N. Akhtar, A. Jamil, S. Aslam, G. Abbas. Appl. Surf. Sci. 334 (2015) 145-149.

6. Y. Zhu, F. Deng, L. Feng, H. Ding, S. Ismat Shah, C. Ni. J. Alloy. Compd. 607 (2014) 132-136.

7. $\quad$ S. Lan, L. Liu, R. Li, Z. Leng, S. Gan. Ind. Eng. Chem. Res. 53 (2014) 3131-3136.

8. P. Cai, H. Song, L. Zhang, Y. Lv. Sensor. Actuat. B: Chem. 173 (2012) 93-98.

9. M.A. Kanjwal, F.A. Sheikh, N.A.M. Barakat, X. Li, H.Y. Kim, I.S. Chronakis. Appl. Surf. Sci. 258 (2012) 3695-3699.

10. H. Lu, S. Wang, L. Zhao, J. Li, B. Dong, Z. Xu. J. Mater. Chem. 21 (2011) 4228-4232.

11. F. Shi, C. Xue. CrystEngComm 14 (2012) 4173-4177.

12. X. Cai, F. Wang, D. Yan, Z. Zhu, X. Gu. Ceram. Int. 40 (2014) 12293-12298.

13. R.K. Jamal, M.A. Hameed, K.A. Adem. Mater. Lett. 132 (2014) 31-34.

14. Y.S. Zou, H. Yang, H.P. Wang, D. Lou, C.J. Tu, Y.C. Zhang. Physica B: Condensed Matter. 414 (2013) 7-12.

15. T. Dedova, M. Krunks, I. Oja Acik, D. Klauson, O. Volobujeva, A. Mere. Mater. Chem. Phys. 141 (2013) 69-74.

16. D. Klauson, I. Gromyko, T. Dedova, N. Pronina, M. Krichevskaya, O. Budarnaja, I. Oja Acik, O. Volobujeva, I. Sildos, K. Utt. Mater. Sci. Semicon. Process 31 (2015) 315-319.

17. X.H. Lu, D. Wang, G.R. Li, C.Y. Su, D.B. Kuang, Y.X. Tong. J. Phys. Chem. C 113 (2009) 13574-13579.

18. J.F. Lei, Z.W. Wang, W.S. Li. Thin Solid Films 573 (2014) 74-78.

19. M. Sabbaghan, A.S. Shahvelayati, S.E. Bashtani. Solid State Sci. 14 (2012) 1191-1195.

20. Y. Shi, C. Zhu, L. Wang, C. Zhao, W. Li, K.K. Fung, T. Ma, A. Hagfeldt, N. Wang. Chem. Mater. 25 (2013) 1000-1006.A.Khorsand Zak, W.H.a. Majid, H.Z. Wang, R. Yousefi, A. Moradi Golsheikh, Z.F. Ren. Ultrason. Sonochem. 20 (2013) 395-400.

21. H. Zhang, R. Wu, Z. Chen, G. Liu, Z. Zhang, Z. Jiao. CrystEngComm 14 (2012) 1775-1779. 
22. N.T. Khoa, S.W. Kim, D. Van Thuan, D.-H. Yoo, E.J. Kim, S.H. Hahn. CrystEngComm 16 (2014) 1344-1348.

23. P.V. Adhyapak, S.P. Meshram, I.S. Mulla, S.K. Pardeshi, D.P. Amalnerkar. Mater. Sci. Semicon. Process 27 (2014) 197-202.

24. M. Navaneethan, J. Archana, Y. Hayakawa. CrystEngComm 15 (2013) 8246-8249.

25. Y. Sun, R. Zou, W. Li, Q. Tian, J. Wu, Z. Chen, J. Hu. CrystEngComm 13 (2011) 6107-6110.

26. M. Wang, B. Zhao, S. Xu, L. Lin, S. Liu, D. He. Chem. Commun. 50 (2014) 930-933.

27. Z.X. Yang, W. Zhong, C. Au, J.Y. Wang, Y.W. Du. CrystEngComm 13 (2011) 1831-1836.

28. Z. Wang, X. Zhou, Z. Li, Y. Zhuo, Y. Gao, Q. Yang, X. Li, G. Lu. RSC Adv. 4 (2014) 23281-23285.

29. Y. Xiao, Y. Lei, B. Zheng, L. Gu, Y. Wang, D. Xiao. RSC Adv. 5 (2015) 21604-21609.

30. P. Zhu, J. Zhang, Z. Wu, Z. Zhang. Cryst. Growth Des. 8 (2008) 3148-3152.

31. T. Laga, L. Ye, R. Hua, G. Liangfa. Mater. Lett. 112 (2013) 5-8.

32. H. Yu, H. Fan, X. Wang, J. Wang. Optik 125 (2014) 1461-1465.

33. S. Das, K. Dutta, A. Pramanik. CrystEngComm 15 (2013) 6349-6353.

34. Y. Sun, L. Wang, X.G. Yu, K.Z. Chen, CrystEngComm 14 (2012) 3199-3203. 